\title{
Preparation and in vitro characterization of 9-nitrocamptothecin-loaded long circulating nanoparticles for delivery in cancer patients
}

This article was published in the following Dove Press journal:

International Journal of Nanomedicine

2 July 2010

Number of times this article has been viewed

\author{
Katayoun Derakhshandeh' \\ Marzieh Soheili' \\ Simin Dadashzadeh ${ }^{2}$ \\ Reza Saghiri ${ }^{3}$ \\ 'Department of Pharmaceutics, \\ Faculty of Pharmacy, University \\ of Medical Science, Kermanshah \\ 67I45-I673, Iran; ${ }^{2}$ Department of \\ Pharmaceutics, Faculty of Pharmacy, \\ Shaheed Beheshti University of \\ Medical Science, Tehran, Iran; \\ ${ }^{3}$ Deptartment of Biochemistry, \\ Pasteur Institute, Tehran, Iran
}

Correspondence: Katayoun Derakhshandeh Department of Pharmaceutics, Faculty of Pharmacy, University of Medical Science, Kermanshah 67|45-1673, Iran

Tel 0098 83| 4276485

Fax 0098 83। 4276496

Email kderakhshandeh@kums.ac.ir

\begin{abstract}
The purpose in this study was to investigate poly(ethylene glycol)-modified poly (d,1-lactide-co-glycolide) nanoparticles (PLGA-PEG-NPs) loading 9-nitrocamptothecin (9-NC) as a potent anticancer drug. $9-\mathrm{NC}$ is an analog of the natural plant alkaloid camptothecin that has shown high antitumor activity and is currently in the end stage of clinical trial. Unfortunately, at physiological $\mathrm{pH}$, these potent agents undergo a rapid and reversible hydrolysis with the loss of antitumor activity. Previous researchers have shown that the encapsulation of this drug in PLGA nanoparticles could increase its stability and release profile. In this research we investigated PLGA-PEG nanoparticles and their effect on in vitro characteristics of this labile drug. 9-NC-PLGA-PEG nanoparticles with particle size within the range of $148.5 \pm 30 \mathrm{~nm}$ were prepared by a nanoprecipitation method. The influence of four different independent variables (amount of polymer, percent of emulsifier, internal phase volume, and external phase volume) on nanoparticle drug-loading was studied. Differential scanning calorimetry and X-ray diffractometry were also evaluated for physical characterizing. The results of optimized formulation showed a narrow size distribution, suitable zeta potential (+1.84), and a drug loading of more than $45 \%$. The in vitro drug release from PLGA-PEG NPs showed a sustained release pattern of up to 120 hours and comparing with PLGA-NPs had a significant decrease in initial burst effect. These experimental results indicate that PLGA-PEG-NPs (versus PLGA-NPs) have a better physicochemical characterization and can be developed as a drug carrier in order to treat different malignancies.
\end{abstract}

Keywords: long circulating nanoparticle, 9-nitrocamptothecin, in vitro characterization

Camptothecin (CPT) and its derivatives play an important role in the treatment of different malignancies. ${ }^{1,2}$ They all have a unique mechanism of action, targeting the nuclear enzyme of DNA topoisomerase I (topo I). CPT inhibits this enzyme by blocking the rejoining step of the cleavage/relegation reaction of topo I, resulting in accumulation of a covalent reaction intermediate, the cleavage complex. The primary mechanism of cell killing by CPT is S-specific killing through potentially lethal collision between advancing replication forks and topo-I cleavable complexes. ${ }^{3,4,5}$ In the structure of these analogues, common features include an aromatic 5-ring system and a lactone moiety which is crucial for their antitumor activity. ${ }^{4}$

9-Nitrocamptothecin (9-NC) is a novel, lipophilic derivative of CPT which has a high antitumor activity which has been used in several clinical trial studies in order to treat advanced pancreatic carcinoma, ovarian epithelial cancer and leukemia. ${ }^{2}$ Unfortunately, the lactone form depends on the $\mathrm{pH}$ of the medium and easily inverts to carboxylate form in alkaline $\mathrm{pHs}$, especially when exposed to serum albumin proteins, which remove its antitumor properties (see Figure 1)., 
A

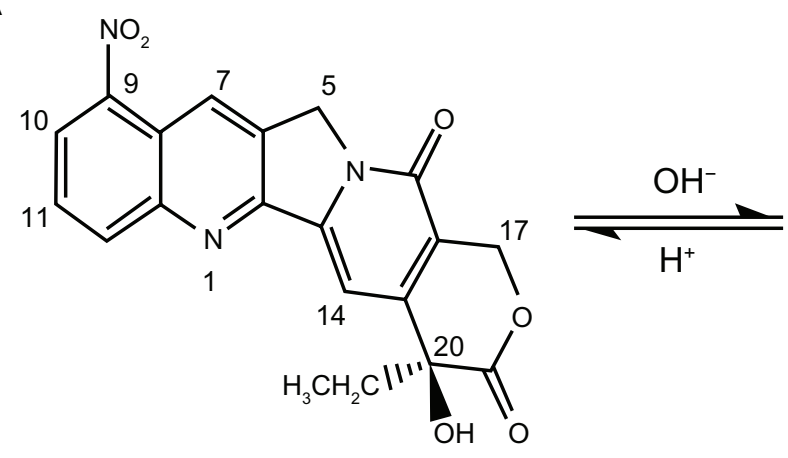

B

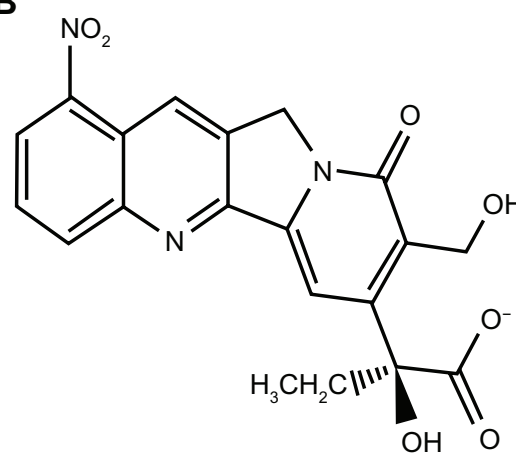

Figure I Chemical structure of lactone and carboxylate forms of 9-nitrocamptothecin showing their equilibrium reaction, (A) lactone form, (B) carboxylate form.

Because of instability at biological $\mathrm{pH}$ and low water solubility, the delivery of lipophilic derivatives like 9-NC is quite challenging. ${ }^{7}$ To eradicate these problems, drug delivery systems such as liposomes, nanocapsules, micellar systems, and conjugates have been used to overcome the cellular resistance and take active pharmacologic agents to the target tissue, increasing bioavailability and drug affect in target tissue, solubilizing for intravascular delivery, and improving the stability of therapeutic agents for $\mathrm{pH}$ hydrolysis and enzymatic degradation. ${ }^{8,9}$

Polymeric nanoparticles composed of PLGA as a biocompatible and biodegradable compound have more stability and lower toxicity compared to other drug delivery systems like solid lipid nanoparticles (SLNs). ${ }^{7}$ But since these structures can be easily opsonized by the mononuclear phagocytic system (MPS) due to their lipophilic surface, they have a short half-life with an in vivo failed function. To address this burden, some hydrophilic conjugates are used to prepare the solution, which have a hydrophilic surface with a lipophilic core in the center called the nanoparticle core shell effect. ${ }^{10-14}$

The most common formulations applied in these polymers are composed of PEG structures named copolymers; the purpose of these PEG chains is to create a barrier layer to block the adhesion of opsonins present in the blood serum, so that the particles can remain camouflaged or invisible to phagocytic cells. ${ }^{15,16}$

PEGylation simply refers to the decoration of a particle surface by covalently grafting, entrapping, or adsorbing PEG chains. Also, in the case of biodegradable nanoparticles, PEG chains can be incorporated as copolymers throughout the particle so that some surface PEG chains are always available even after the degradation of surface layers. ${ }^{15-17}$
Other important points in the application of these copolymers are controlled initial release and smaller size compared to nanoparticles containing PLGA polymers which leads to increased in vivo stability and effect. ${ }^{17-22}$

Copolymeric nanoparticles have different characteristics in that the formation of each of them depends on the copolymer composition, molecular geometry, the length of the chain, and the method of preparation. ${ }^{23-26}$

The aim in present study was to prepare 9-NC loaded nanoparticles by PLGA-PEG copolymer and compare the physicochemical properties results with a previous study which involved the use of PLGA polymer. ${ }^{7}$ The resulting 9-NC nanoparticles were characterized with regard to morphology, size, drug loading, and in vitro drug release.

\section{Materials and methods Materials}

9-Nitrocamptothecin (9-NC), 99.8\% pure, was purchased from Yuanjian Pharmaceutical Technology Develop Co., (China). Poly(d, 1-lactide-co-glycolide) (PLGA; 50:50 Mw 12000 , inherent viscosity of $0.16-0.24 \mathrm{dl} / \mathrm{g}$ ) and PLGA-PEG $5 \%(\mathrm{Mw}$ 12000) were obtained from Bohringer Ingelheim Co. (Germany). Polyvinyl alcohol (PVA; Mw 30,000 Da, 87\% hydrolyzed) was a gift from Mowiol (Germany). Analytical grade acetone and acetonitrile were obtained from Merck (Darmstadt, Germany). All other chemical reagents used were of pharmaceutical grade.

\section{Preparation of nanoparticles}

The nanoprecipitation method was employed for the formation of drug-encapsulated PLGA-PEG nanoparticles similarly to previously performed study. ${ }^{7}$ Briefly, PLGA-PEG was dissolved in acetone. Then the copolymer solution containing exact amount of drug was added 

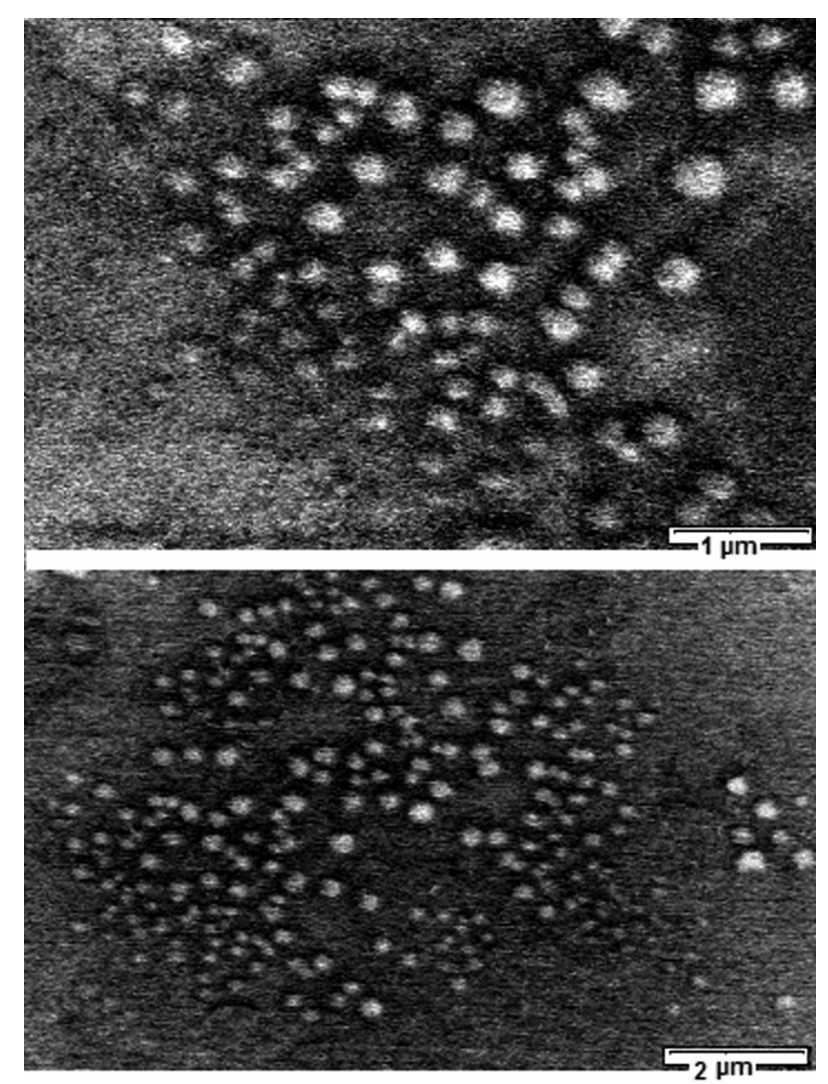

Figure 2 Scanning table electron micrograph of 9-nitrocamptothecin nanoparticles.

drop-wise $(0.5 \mathrm{~mL} / \mathrm{min})$ into PVA aqueous solution $(\mathrm{pH}$ was adjusted to 3 by $0.1 \mathrm{~N} \mathrm{HCl}$ ) and stirred magnetically at room temperature until complete evaporation of the organic solvent. Next, the nanoparticle suspension was centrifuged by ultracentrifuge (XL-90 Beckman, Brea, CA) at $40,000 \mathrm{rpm}$ and $4^{\circ} \mathrm{C}$ for 90 minutes. The separated nanoparticles were redispersed and centrifuged three times in distilled water $(\mathrm{pH} 3)$ in order to remove free drug and excess surfactant completely. The acidity of the medium used in this process was because of stabilizing the lactone form of 9-nitrocamptothecin. Finally, nanoparticles were dried via desiccator at room temperature for 24 hours, and then were characterized.

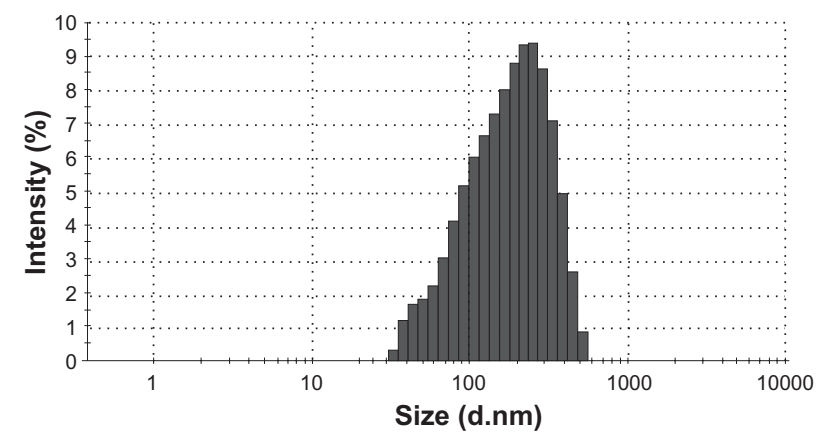

Figure 3 Size distribution of 9-NC nanoparticles.
In this study, we evaluated the effects on drug loading of four different variable amounts of polymer, percent of emulsifier, internal phase volume, and external phase volume, to approach optimum formulation by one at a time statistical method.

\section{Characterization of 9-NC Determination of particle size}

The mean size and zeta potential of nanoparticles were measured by photon correlation spectroscopy (PCS) (SEMATech), at a scattering angle of $90^{\circ}$ and at a temperature of $25^{\circ} \mathrm{C}$ using samples appropriately diluted with super pure water.

\section{Particle morphology}

The morphology of nanospheres was observed by scanning electron microscopy (SEM) (Philips, the Netherlands). Samples were coated with a thin layer of colloidal gold applied in a cathodic vacuum evaporator before observation by SEM at $20 \mathrm{KV}$.

\section{Differential scanning calorimetry (DSC)}

The physical state of the nanoparticle components was characterized by thermal analysis in DSC-60 analyzer (Shimadzu Co., Kyoto, Japan). Samples (10 mg) were sealed into aluminum pans and heated in an inert atmosphere of nitrogen with a heat rate of $10^{\circ} \mathrm{C} / \mathrm{min}$. Calibration of the system was performed using octadecane and indium.

\section{X-ray diffractometry}

An X-ray diffractometer, (XRD; Philips APD), was used for diffraction studies. The samples were exposed to $\mathrm{CuK} \alpha$ radiation $(40 \mathrm{kv}, 20 \mathrm{~mA})$ and scanned from $2^{\circ}$ to $70^{\circ}, 2 \theta$ at a step size of $0.02^{\circ}$, and step time of $5 \mathrm{~S}$. Samples used for XRD analysis were exactly the same as those used for DSC analysis.

\section{Determination of drug content}

Drug content was determined using a validated high-pressure liquid chromatography (HPLC) method. ${ }^{7}$ Nanoparticles were dissolved in acetonitrile $(2 \mathrm{~mL})$ and sonicated for 2 minutes. The organic solvent was evaporated until the stream of nitrogen gas and then was dissolved in $1.5 \mathrm{~mL}$ mobile phase of HPLC assay.

The 1001 HPLC was equipped with an ultraviolet detector model 2700 at $370 \mathrm{~nm}$ (Knauer, Germany) and a column 
of Nucleosil-100 C18 with the mobile phase containing a mixture of acetonitrile-water (43:57, V/V, pH 3).

The amount of drug loading in nanoparticles was calculated using the following equation:

$$
\begin{aligned}
& \text { Encapsulation } \\
& \text { efficiency }(\%)
\end{aligned}=\frac{\begin{array}{l}
\text { (Am nount of 9-NC } \\
\text { in (Amount of 9-NC used } \\
\text { in formulation) }
\end{array}}{100}
$$

\section{In vitro drug release}

The in vitro drug release of nanoparticles was determined by dialysis method using Franz diffusion cell. A total of $2 \mathrm{~mL}$ of NP suspension in phosphate-buffered saline (PBS, 0.1M, pH 7.4) was placed in donor site and $25 \mathrm{~mL}$ PBS in receptor part incubated at $37^{\circ} \mathrm{C}$ under magnetic stirring (200 rpm). At specific time intervals, $500 \mu \mathrm{L}$ of medium was taken and replaced with the same volume of fresh PBS. The taken samples were acidified with 5\% perchloric acid before UV analysis to evaluate the total form of 9-NC (lactone and carboxylate forms). The obtained release data were evaluated by zero order, first order and Higuchi equations to assay the release kinetics. To select the best model, the relevant correlation coefficients were considerable.

\section{Statistical analysis}

The experiments were repeated three times and the results were expressed as mean \pm standard deviation. Statistical analysis was undertaken using Student's $t$ test and $P$ value $<0.05$ were considered statistically significant.

\section{Results and discussion}

Carrier-mediated drug delivery offers a number of design opportunities to tailor a delivery route around the details of particular drugs, including their modes of action and potential side effects. ${ }^{27,28}$ Such carrier functions can be quite diverse, including transport to the targeted tissue, control of intracellular distribution, and protection against degradation or elimination by the mononuclear phagocytic systems (MPS). ${ }^{29,30}$ For instance, it has been demonstrated that Paclitaxel nanoparticles have a long time release accompanied with a small size which is so important for phagocytic systems. ${ }^{15,31-33}$ Also preparing NPs of tumor necrosis factor- $\alpha$ (TNF- $\alpha)$, cisplatin, indomethacin, iboprufen, and so many other drugs has shown that these carriers can deliver the drug to the tissue in the best way with the least side effects and a long time circulation in the body. ${ }^{22,34,35}$ All these features prompted us to investigate the possibility of developing these nanoparticles as a controlled release drug formulation. In the development of new colloidal structures as circulating carriers, the assessment of their performance as drug carriers necessarily comprises the knowledge of the loaded amount of drug, its physical state and distribution inside the carrier, as well as its release kinetics and the influence of environmental factors on it.

In our previous study, ${ }^{7}$ PLGA-loaded 9-NC nanoparticles were prepared by nanoprecipitation method and four different independent variables on the characteristics of nanoparticles were evaluated. The results of optimized formulations showed a narrow size distribution with a polydispersity index of $0.01 \%$, an average diameter of $207 \pm 26 \mathrm{~nm}$, and a drug loading of more than $30 \%$. The in vitro drug release profile showed a sustained 9-NC release up to 160 hours indicating the controlled release of 9-NC via nanoparticles.

But, since PLGA nanoparticles can be uptaken by phagocytic systems easily and have a rapid initial release (15\%-20\% in first 5 hours), in this study, we used PLGAPEG for preparing nanoparticles and characterized them

Table I Results of drug loadings according to amount of polymer $(n=3)$

\begin{tabular}{llllll}
\hline Formulation & $\begin{array}{l}\text { Polymer } \\
(\mathbf{m g})\end{array}$ & $\begin{array}{l}\text { PVA } \\
(\mathbf{m g})\end{array}$ & $\begin{array}{l}\text { Int. phase } \\
(\mathbf{m L})\end{array}$ & $\begin{array}{l}\text { Ext. phase } \\
(\mathbf{m L})\end{array}$ \\
\hline 1 & 50 & 50 & 12 & 22 & $13.5 \pm 3.7$ \\
2 & 100 & 50 & 12 & 22 & $15.6 \pm 2.7$ \\
3 & 125 & 50 & 12 & 22 & $22.3 \pm 3.4$ \\
4 & 150 & 50 & 12 & 22 & $28.7 \pm 2.3$ \\
5 & 165 & 50 & 12 & 22 & $45.3 \pm 2.9$ \\
6 & 180 & 50 & 12 & 22 & $33.3 \pm 2.1$ \\
7 & 200 & 50 & 12 & 22 & $31.3 \pm 4.8$ \\
8 & 250 & 50 & 12 & 22 & $28.9 \pm 3.1$ \\
9 & 300 & 50 & 12 & 22 & $26.5 \pm 1.9$ \\
\hline
\end{tabular}

Abbreviations: EE, entrapment efficacy; PVA, polyvinyl alcohol. 
Table 2 Results of drug loadings according to amount of surfactant $(n=3)$

\begin{tabular}{llllll}
\hline Formulation & $\begin{array}{l}\text { Polymer } \\
(\mathbf{m g})\end{array}$ & $\begin{array}{l}\text { PVA } \\
(\mathbf{m g})\end{array}$ & $\begin{array}{l}\text { Int. phase } \\
(\mathbf{m L})\end{array}$ & $\begin{array}{l}\text { Ext. phase } \\
(\mathbf{m L})\end{array}$ \\
\hline 1 & 165 & 0 & 12 & 22 & - \\
2 & 165 & 25 & 12 & 22 & $18.2 \pm 5.8$ \\
3 & 165 & 35 & 12 & 22 & $23.4 \pm 6.2$ \\
4 & 165 & 50 & 12 & 22 & $45.3 \pm 2.9$ \\
5 & 165 & 75 & 12 & 22 & $28.8 \pm 4.9$ \\
6 & 165 & 100 & 12 & 22 & $15.2 \pm 4.2$ \\
\hline
\end{tabular}

Abbreviations: EE, entrapment efficacy; PVA, polyvinyl alcohol.

to be taken to the next phase of study like cytotoxicity and in vivo studies.

\section{Preparation of nanoparticles}

First formulations showed that drug amounts more than $1 \mathrm{mg} / \mathrm{mL}$ had yellow sedimentation and loading efficiency was not increased by adding amounts of drug and supernatant, indicating that nanoparticles could not accept amounts more than $1 \mathrm{mg} / \mathrm{mL}$.

Several formulations were prepared and four variables were considered, and the drug loadings were obtained to identify the best loading in order to select one optimum formulation and to characterize the other physical assessments on the optimum one (Tables 1-4).

In comparing the results of calculated loadings, increasing the amount of polymer has a marked effect on drug loading (Table 1).

Amount of the emulsifier has no significant effect on encapsulation of drug but it is necessary for nanoparticle preparation (Table 2). The volume of internal phase and external phase has a reversal effect on drug loading after volumes of $12 \mathrm{~mL}$ and $22 \mathrm{~mL}$, respectively (Tables 3 and 4).

As the results show, the optimum formulation is the one consisting of $165 \mathrm{mg}$ polymer, $50 \mathrm{mg}$ PVA, $12 \mathrm{~mL}$, and $22 \mathrm{~mL}$ internal and external phases, respectively, which had a drug content of more than $45 \%$.

The PLGA nanoparticle which is used as control group in comparison of physicochemical characteristics was achieved by $165.27 \mathrm{mg}$ of polymer, $1.31 \%$ of emulsifier, $21.33 \mathrm{~mL}$ external phase, and $12 \mathrm{~mL}$ internal phase volumes. The resulting 9-NC PLGA nanoparticles showed the response to size with average of $207 \mathrm{~nm}$ and drug loading of about $33 \%{ }^{7}$

\section{Nanoparticle characterization}

The morphology of the optimized PLGA-PEG nanoparticles examined by SEM was spherical as shown in Figure 4. Average size of nanoparticles was found to be around $148.5 \pm 30 \mathrm{~nm}$ with a polydispersity of $0.07 \%$. It is concluded that the steric barrier of PEG shell could be the main cause to avoid aggregation of internal phase droplets in preparation pathway; therefore PLGA-PEG nanoparticles have a smaller size than PLGA ones. The zeta potential of the nanoparticles was about $+1.84 \mathrm{mV}$ over what is suitable for stable colloidal dispersion of nanoparticles.

The DSC thermograms of 9-NC, PLGA-PEG copolymer, physical mixture of drug and polymer, and 9-NC nanoparticles are shown in Figure 5. The DSC curve of 9-NC showed a single melting peak which started to degrade as it melted. The PLGA-PEG thermogram displayed an endothermic peak investigative of polymer transition temperature $(\mathrm{Tg})$. No 9-NC melting peak was visible in the case of drug-loaded nanoparticles. This might be due to the amorphous state of the drug when dispersed in the nanoparticles. Since the curve of copolymer has not any shift in Tg, it is concluded that there is no occurrence of prominent interaction between the drug and copolymer.

Table 3 Results of drug loadings according to internal phase volume $(n=3)$

\begin{tabular}{llllll}
\hline Formulation & $\begin{array}{l}\text { Polymer } \\
(\mathbf{m g})\end{array}$ & $\begin{array}{l}\text { PVA } \\
(\mathbf{m g})\end{array}$ & $\begin{array}{l}\text { Int. phase } \\
(\mathbf{m L})\end{array}$ & $\begin{array}{l}\text { Ext. phase } \\
(\mathbf{m L})\end{array}$ \\
\hline 1 & 165 & 50 & 5 & 22 & - \\
2 & 165 & 50 & 10 & 22 & $35.89 \pm 4.2$ \\
3 & 165 & 50 & 12 & 22 & $45.3 \pm 2.9$ \\
4 & 165 & 50 & 15 & 22 & $23.4 \pm 4.7$ \\
5 & 165 & 50 & 25 & 22 & $11.7 \pm 4.3$ \\
\hline
\end{tabular}


Table 4 Results of drug loadings according to amount of external volume $(n=3)$

\begin{tabular}{llllll}
\hline Formulation & $\begin{array}{l}\text { Polymer } \\
(\mathbf{m g})\end{array}$ & $\begin{array}{l}\text { PVA } \\
(\mathbf{m g})\end{array}$ & $\begin{array}{l}\text { Int. phase } \\
(\mathbf{m L})\end{array}$ & $\begin{array}{l}\text { Ext. phase } \\
(\mathbf{m L})\end{array}$ \\
\hline 1 & 165 & 0 & 12 & 5 & - \\
2 & 165 & 25 & 12 & 10 & $10.1 \pm 6.9$ \\
3 & 165 & 35 & 12 & 20 & $13.1 \pm 5.2$ \\
4 & 165 & 50 & 12 & 22 & $45.3 \pm 2.9$ \\
5 & 165 & 75 & 12 & 25 & $38.3 \pm 7.4$ \\
6 & 165 & 100 & 12 & 30 & $30.0 \pm 8.6$ \\
\hline
\end{tabular}

Regarding physical mixture, there is not any peak at the melting point of 9-NC. According to glass liquid transition of PLGA-PEG which is at lower temperatures, it is possible that at higher temperatures molecular dispersion of drug in polymer occurs during DSC process.

XRD patterns were used to confirm these results, and these are shown in Figure 5. As can be seen, the curves of 9-NC exhibit a sharp peak at about $2 \theta$ scattered angle 27 indicating the crystalline nature of 9-NC. This kind of trait was absent in pattern of nanoparticles investigating that $9-\mathrm{NC}$ was not in crystalline form in nanoparticles.

\section{In vitro drug release}

The 9-NC release profiles from optimum PLGA-PEG nanoparticles are shown in Figure 6. The procedure was performed using the dialysis technique using a dialysis membrane having a molecular weight cutoff of 12,000 Da (Sigma, St. Louis, MO) which was fixed on Franz diffusion cell and donor and acceptor medium was PBS ( $\mathrm{pH}$ 7.4). In the release curve, two parts are seen. The first part shows the initial phase of release which has a rapid trend during the first 5 hours, followed by a distinct prolonged release for more than 120 hours. The rapid initial release can be due to a part of drug on the surface of nanoparticles and the delayed part is thought to be because of

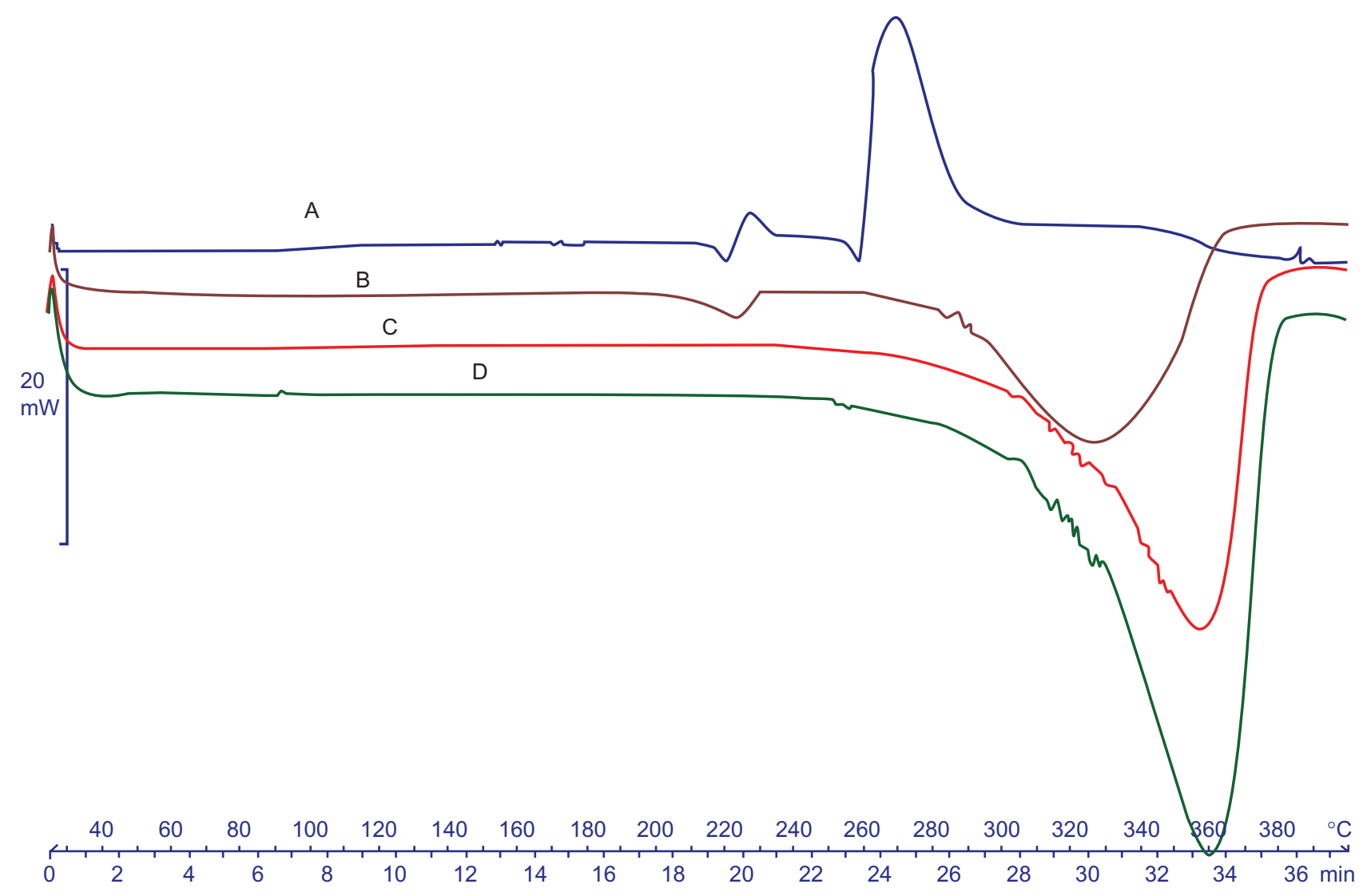

Figure 4 DCS thermograms obtained for A) 9-NC, B) Physical mixture, C) Nanoparticles, D) Copolymer. 


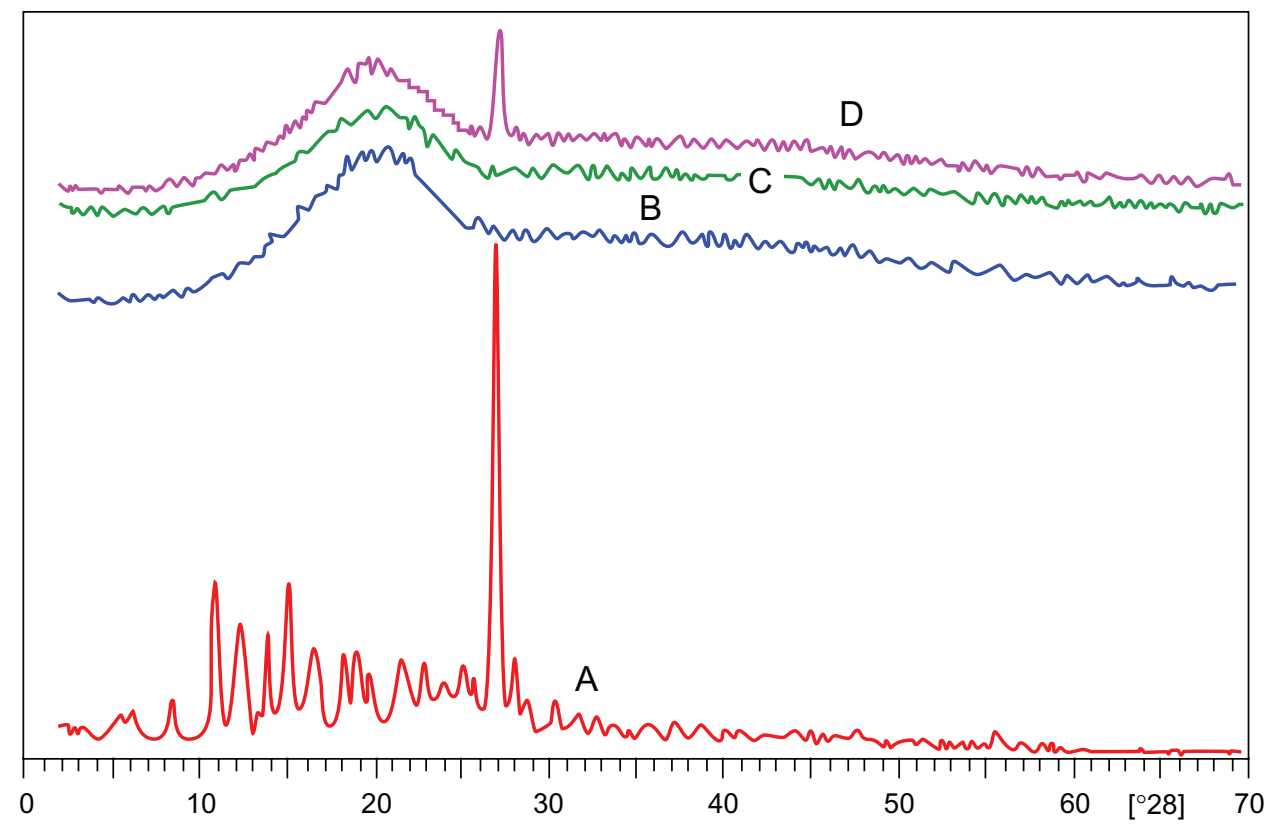

Figure 5 XRD patterns. A) 9-NC, B) Copolymer, C) Nanoparticle, D) Physical mixture of drug and copolymer.

diffusion of dissolved drug through polymeric matrix and its exit to the dissolution medium, where as in our previous study about $20 \%$ of the drug was released over a period of 20 hours, followed by an extended release period of more than 160 hours (see Figure 6). This difference of release profile could be explained by presence of the hydrophilic PEG shell around the nanoparticles, causing increased diffusion of water to the core of particles. On the other hand, compared to plain PLGA nanoparticles, the hydrophilic surface decreases adsorption of lipophilic drugs on the surface of nanoparticles and decreases initial burst drug release.

Release kinetics was evaluated by calculating data via zero order, first order, and Higuchi equations. According to the results, the drug release followed the Higuchi equation and related correlation coefficients were better than both zero and first order kinetics (Table 5).

Two possible mechanisms according to the Higuchi model may be involved in the release of 9-NC from nanoparticles; the dissolution diffusion of the drug from the matrices and matrix erosion resulting from degradation of polymer.

Baker and Lonsdale developed the equation: 2/3[1-(1$\mathrm{Q})^{2 / 3} \mathrm{~J}-\mathrm{Q}=\mathrm{k} . \mathrm{t}$, to describe the release of drug incorporated

Table 5 Results of release kinetics according to zero order, first order, and Higuchi equations

\begin{tabular}{llll}
\hline $\mathrm{K}_{\mathrm{h}}^{\mathrm{a}}$ & \multicolumn{3}{l}{ Regression coefficient $\left(\boldsymbol{R}^{2}\right)$} \\
\cline { 2 - 2 }$/ \mathrm{h}^{0.5}$ & Higuchi & First order & Zero order \\
\hline $4.35 \mathrm{I}$ & 0.989 & 0.922 & 0.896 \\
\hline
\end{tabular}

in a spherical matrix based on a diffusion mechanism. ${ }^{35}$ By fitting the observed data, shown in Figure 6, to the spherical matrix model, correlation coefficients of between 0.956 and 0.994 were obtained.

The Hixson-Crowell cube root model, $(100-\mathrm{Q} . \mathrm{t})^{1 / 3}=100^{1 / 3}-\mathrm{K}_{\mathrm{HC}} \cdot \mathrm{t}$, where $\mathrm{K}_{\mathrm{HC}}$ is the Hixson-Crowell rate constant, describes the release from the systems, where it depends on the change in surface area and diameter of the particles with time and mainly applies in case of systems, which dissolve or erode over time. ${ }^{36}$ By fitting the release data to this model, correlation coefficients of $0.880-0.937$ were obtained.

These results indicate that the release of $9-\mathrm{NC}$ from nanoparticles may be more consistent with a diffusion mechanism than a matrix erosion mechanism. In similar studies, Gao and coworkers prepared different block copolymer micelles of 9-NC, amphiphilic copolymers

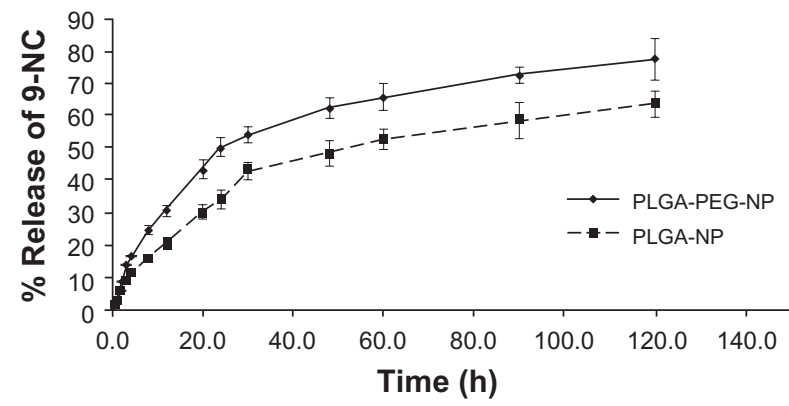

Figure 6 9-NC release curve from optimum formulation of PLGA-PEG and PLGA nanoparticles. 
of methoxy poly(ethylene glycol)-b-poly(D,L-lactide), and copolymers derived from d,l-lactide, trimethylene carbonate, and methylated poly(ethylene glycol). ${ }^{37,38}$ These micelles could significantly increase drug solubility; release of 9-NC was nearly zero order after the burst release, and the long hydrophobic chain length led to slower release rate about $50 \%$ over 60 hours, similar to results gained in our experiment. ${ }^{37,38}$

In general, in vitro release profiles shows that these nanoparticles have the capacity to successfully release 9-NC using a sustained rate.

\section{Conclusion}

In this investigation, PLGA-PEG nanoparticles loaded with 9-NC (a novel potent anticancer drug) were prepared by nanoprecipitation, and the physicochemical characteristics included an investigation of their properties, such as drug loading, morphology, size, and thermal behavior were evaluated and compared with the results of our previous study, in which these NPs were prepared using PLGA polymer instead of PLGA-PEG copolymer. The images of SEM indicated the optimized NPs were spherical in shape without any aggregation or adhesion. More detailed studies showed that the particle size of NPs through using PLGA-PEG is smaller than PLGA samples. Drug loading was much higher than the previous study, and the initial release was slower. But as mentioned above the total release was faster than PLGA NPs, which can be explained by the hydrophilic characteristic of PLGA-PEG copolymer. This point causes a rapid release compared with PLGA that is a lipophil polymer and can keep the drug longer than PLGAPEG copolymer.

Overall physical evaluation comparison shows that PLGA-PEG nanoparticles can be a promising controlled release delivery system of lipophilic drugs like 9-NC specially in cancer therapies which need an acute and target treatment during therapeutically period.

However, the in vivo behavior of drug release, the speed of clearance, and final biodistribution of nanoparticles also need to be confirmed in future studies.

\section{Acknowledgment/disclosure}

The authors would like to thank Miss Parvaneh Abbasian (Biopharmacy Laboratory, Shaheed Beheshti Medical University, Iran) for her kind assistance with the HPLC analysis. This work was supported financially by the Faculty of Pharmacy, University of Medical Science, Kermanshah, Iran.

\section{References}

1. Panaym J, Labhasetwar V. Biodegradable nanoparticles for drug and gene delivery to cells and tissue. Adv Drug Deliv Rev. 2003;55:329-347.

2. Garcia-Carbonero R, Supko JG. Current perspectives on the clinical experience, pharmacology and continued development of the camptothecins. Clin Cancer Res. 2002;8:641-661.

3. Iyer L, Ratain MJ. Clinical pharmacology of camptothecins. Cancer Chem Pharm. 1998;42:S31-S34.

4. Erickson-Miller CL, May RD, Tomaszewski J, et al. Differential toxicity of camptothecin, topotecan and 9-aminocamptothecin to human, canine, and murine myeloid progenitors (CFU-GM) in vitro. Cancer Chem Pharm. 1997;39:467-472.

5. Liu LF, Desai SD, Li TK, Mao Y, Sun M, Sim SP. Mechanism of action of camptothecin. Ann N Y Acad Sci. 2000;922:1-10.

6. Fassberg J, Stella VJ. A kinetic and mechanistic study of the hydrolysis of camptothecin and some analogues. J Pharm Sci. 1992;81: 676-684.

7. Derakhshandeh K, Dadashzadeh S, Erfan M. Encapsulation of 9-nitrocamptothecin, a novel anticancer drug, in biodegradable nanoparticles: Factorial design, characterization and release kinetics. Eur J Pharm biopharm. 2007;66:34-41.

8. Lu W, Han X, Liu J, et al. 9- NC loaded folate-conjugated polymer micelles as tumor targeted drug delivery systems: preparation and evaluation in vitro. Int J Pharm. 2009;372:125-131.

9. Lu JL, Wang JC, Zhao SX, et al. Self-microemulsifying drug delivery system (SMEDDS) improves anticancer effect of oral 9-nitrocamptothecin on human cancer xenograft in nude mice. Eur J Pharm Biopharm. 2008;69:899-907.

10. Arbuk SG, Takimoto $\mathrm{CH}$. An overview of topoisomerase I targeting agents. Semin Hematol. 1998;35:3-12.

11. Dalwadi G, Sunderland B. An ion pairing approach to increase the loading of hydrophilic and lipophilic drugs into PEGylated PLGA nanoparticles. Eur J Pharm Biopharm. 2009;71(2):231-242.

12. Beletsi A, Panagi Z, Avgoustakis K. Biodistribution properties of nanoparticles based on mixtures of PLGA with PLGA-PEG diblock copolymers. Int J Pharm. 2005;298:233-241.

13. Yan X, Scherphof GL, Kamps JAAM. Liposome opsonization. J Liposome Res. 2005;15:109-139.

14. Gaucher G, Dufresne MH, Sant VP, Kang N, Maysinger D, Leroux JC. Block copolymer micelles: preparation, characterization and application in drug delivery. J Control Release. 2005;109:169-188.

15. Peracchia MT, Harnisch S, Pinto-Alphandary H, et al. Visualization of in vitro protein-rejecting properties of PEGylated stealth polycyanoacrylate nanoparticles. Biomaterials. 1999;20:1269-1275.

16. Owens DE, Peppas NA. Opsonization, biodistribution, and pharmacokinetics of polymeric nanoparticles. Int J Pharm. 2006;307(1):93-102.

17. Porter CJH, Moghimi SM, Illum L, Davis SS. The polyoxyethylene polyoxypropylene block copolymer Poloxamer- 407 selectively redirects intravenously injected microspheres to sinusoidal endothelial-cells of rabbit bone-marrow. FEBS Letter. 1992b;305:62-66.

18. Gref R, Minamitake Y, Peracchia MT, Trubetskoy V, Torchilin V, Langer R. Biodegradable long-circulating polymeric nanospheres. Science. 1994;263(5153):1600-1603.

19. Kwon GS, Okano T. Polymeric micelles as new drug carriers. Adv Drug Deliv Rev. 1996;21:107-116.

20. Xiong XY, Li YP, Li ZL, et al. Vesicles from pluronic/poly (lactic acid) block copolymers as new carriers for oral insulin delivery. $J$ Control Release. 2007;120(1-2):11-7.

21. Fernandez-Carballoido A, Pastoriza P, Barica E, Montejo C, Negro S. PLGA/PEG derivative polymeric matrix for drug delivery system application: characterization and cell viability studies. Int J Pharm. 2008;352:50-57.

22. Letchford K, Burt H. A review of the formulation and classification of amphiphilic block copolymer nanoparticulate structures: micelles, nanospheres, nanocapsules and polymersomes. Eur J Pharm Biopharm. 2007;65(3):259-269. 
23. Paciotti GF, Kingston DGI, Tamarkin L. Colloidal gold nanoparticles: a novel nanoparticle platform for developing multifunctionaltumortargeted drug delivery vectors. Drug Deliv Res. 2006;67:47-54.

24. Klumpp C, Kostarelos K, Prato M, Bianco A. Functionalized carbon nanotubes as emerging nanovectors for the delivery of therapeutics. Biochimica et Biophysica Acta. 2006;1758:404-412.

25. Vonarbourg A, Passirani C, Saulnier P, Benoit JP. Parameters influencing the stealthiness of colloidal drug delivery systems. Biomaterials. 2006;27:4356-4373.

26. Kostarelos K. Rational design and engineering of delivery systems for therapeutics: biomedical exercises in colloid and surface science. Adv Colloid Interface Sci. 2003;106:147-168.

27. Hubbell JA. Enhancing drug function. Science. 2003;300:595-596.

28. Allen TM, Cullis PR. Drug delivery systems: entering the mainstream. Science. 2004;303:1818-1822.

29. Savic R, Luo L, Eisenberg A, Maysinger D. Micellar nanocontainers distribute to defined cytoplasmic organelles. Science. 2003;300: 615-618.

30. Danhier F, Lecouturier N, Vroman B, et al. Paclitaxel-loaded PEGylated PLGA-based nanoparticles: In vitro and in vivo evaluation. J Control Release. 2009;133:11-17.

31. Hu Y, Xie J, Tong YW, Wang CH. Effect of PEG conformation and particle size on the cellular uptake efficiency of nanoparticles with the HepG2 cells. J Control Release. 2007;118(1):7-17.
32. Burt HM, Liggins RT. Polyether-polyester diblock copolymers for the preparation of paclitaxel loaded polymeric micelle formulations. $A d v$ Drug Deliv Rev. 2002;54:191-202.

33. Liu W, Yang A, Yang L, Li Z, Xu H, Yang X. Tumor necrosis factor alpha blocking peptide loaded PEG-PLGA nanoparticles: preparation and in vitro evaluation. Int J Pharm. 2007;331:123-132.

34. Avgoustakis K, Beletsi A, Panagi Z, Klepetsanis P, Karydas AG, Ithakissios DS. PLGAmPEG nanoparticles of cisplatin: in vitro nanoparticle degradation, in vitro drug release and in vivo drug residence in blood properties. J Control Release. 2002;79:123-135.

35. Baker RW, Lonsdale HK. Controlled release of biologically active agent. in: AC Tanquary, R Lacey, eds. New York: Plenum Press. 1974; p. $15-71$.

36. Hixson AW, Crowell JH. Dependence of reaction velocity upon surface and agitation. 1. Theoretical consideration. Ind Eng Chem. 1931;23: 923-931.

37. Gao J, Ming J, Hec B, Fanc Y, Guc Z, Zhang X. Preparation and characterization of novel polymeric micelles for 9-nitro-20(S)-camptothecin delivery. Eur J Pharm Sci. 2008;34:85-93.

38. Gao JM, Ming J, He B, Gu ZW, Zhang XD. Controlled release of 9-nitro-20(S)-camptothecin from methoxy poly(ethylene glycol)poly(D,L-lactide) micelles. Biomed Mater. 2008;3:015013.
International Journal of Nanomedicine

\section{Publish your work in this journal}

The International Journal of Nanomedicine is an international, peerreviewed journal focusing on the application of nanotechnology in diagnostics, therapeutics, and drug delivery systems throughout the biomedical field. This journal is indexed on PubMed Central,

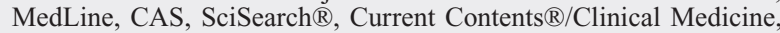

\section{Dovepress}

Journal Citation Reports/Science Edition, EMBase, Scopus and the Elsevier Bibliographic databases. The manuscript management system is completely online and includes a very quick and fair peer-review system, which is all easy to use. Visit http://www.dovepress.com/ testimonials.php to read real quotes from published authors. 